\title{
Cytauxzoon europaeus infections in domestic cats in Switzerland and in European wildcats in France: a tale that started more than two decades ago
}

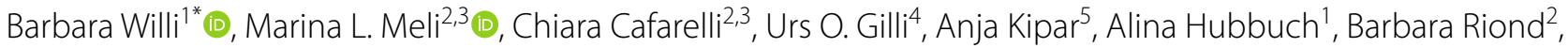 \\ Judith Howard ${ }^{6}$, Daniel Schaarschmidt ${ }^{7}$, Walter Regli ${ }^{8}$ and Regina Hofmann-Lehmann ${ }^{2,3}$ (I)
}

\begin{abstract}
Background: Cytauxzoon spp. infection is believed to be a newly emerging tick-borne disease in felids in Europe, with three species of the haemoparasite having recently been differentiated in wild felids. In Switzerland, rare infections have been documented in domestic cats in the west and northwest of the country, the first of which was in 2014. The aims of the present study were: (i) to characterize a Cytauxzoon spp. hotspot in domestic cats in central Switzerland; (ii) to elucidate the geographic distribution of Cytauxzoon spp. in domestic cats in Switzerland; (iii) to assess suspected high-risk populations, such as stray and anaemic cats; and (iv) to investigate the newly emerging nature of the infection. Cytauxzoon spp. were further differentiated using mitochondrial gene sequencing.
\end{abstract}

Methods: The overall study included samples from 13 cats from two households in central Switzerland (study A), 881 cats from all regions of Switzerland (study B), 91 stray cats from a hotspot region in the northwest of Switzerland and 501 anaemic cats from across Switzerland (study C), and 65 Swiss domestic cats sampled in 2003 and 34 European wildcats from eastern France sampled in the period 1995-1996 (study D). The samples were analysed for Cytauxzoon spp. using real-time TaqMan quantitative PCR, and positive samples were subjected to $18 \mathrm{~S}$ rRNA, cytochrome $b$ (CytB) and cytochrome c oxidase subunit I (COI) gene sequencing.

Results: In study A, six of 13 cats from two neighbouring households in central Switzerland tested postive for Cytauxzoon spp.; two of the six infected cats died from bacterial infections. In studies B and C, only one of the 881 cats (0.1\%; $95 \%$ confidence interval [Cl]: $0-0.3 \%)$ in the countrywide survey and one of the 501 anaemic cats $(0.2 \% ; 95 \%$ Cl: 0-0.6\%) tested postive for Cytauxzoon spp. while eight of the 91 stray cats in the northwest of Switzerland tested positive (8.8\%; 95\% Cl: 3.0-14.6\%). In study D, Cytauxzoon spp. was detected in one of the 65 domestic cat samples from 2003 (1.5\%; 95\% Cl: 0-4.5\%) and in ten of the 34 European wildcat samples from 1995 to 1996 (29\%; 95\% Cl: $14.2-44.7 \%$ ). The isolates showed $\geq 98.6 \%$ sequence identities among the 185 rRNA, CytB and CO/ genes, respectively, and fell in the subclade Cytauxzoon europaeus based on CytB and COI gene phylogenetic analyses.

\footnotetext{
*Correspondence: bwilli@vetclinics.uzh.ch

${ }^{1}$ Clinic for Small Animal Internal Medicine, Department for Small Animals,

Vetsuisse Faculty, University of Zurich, Zurich, Switzerland

Full list of author information is available at the end of the article

Author Walter Regli is deceased.
}

(c) The Author(s) 2022. Open Access This article is licensed under a Creative Commons Attribution 4.0 International License, which permits use, sharing, adaptation, distribution and reproduction in any medium or format, as long as you give appropriate credit to the original author(s) and the source, provide a link to the Creative Commons licence, and indicate if changes were made. The images or other third party material in this article are included in the article's Creative Commons licence, unless indicated otherwise in a credit line to the material. If material is not included in the article's Creative Commons licence and your intended use is not permitted by statutory regulation or exceeds the permitted use, you will need to obtain permission directly from the copyright holder. To view a copy of this licence, visit http://creativecommons.org/licenses/by/4.0/. The Creative Commons Public Domain Dedication waiver (http://creativeco mmons.org/publicdomain/zero/1.0/) applies to the data made available in this article, unless otherwise stated in a credit line to the data. 
Conclusions: The study challenges the newly emerging nature of Cytauxzoon spp. in central Europe and confirms that isolates from domestic cats in Switzerland and European wild felids belong to the same species.

Keywords: Cytauxzoon spp., Cytauxzoon sp., Cytauxzoon felis, Domestic cats, Stray cats, Wild felids, European wildcat, Phylogenetic analysis, 185 rRNA, Prevalence

\section{Background}

Cytauxzoonosis is a tick-borne disease of domestic cats and wild felids that is caused by the apicomplexan haemoparasites Cytauxzoon spp. Cytauxzoon felis is the best-characterized species within this group of pathogens $[1,2]$. Infection generally induces a rapidly progressive disease in domestic cats that is associated with high mortality [3, 4], but survival after clinical infection and subclinical persistent parasitaemia has been documented [5-7]. Since the first report in the mid-1970s [8], C. felis infection has been considered an emerging disease with an expanding case distribution in the USA [9]. Both Dermacentor variabilis and Amblyomma americanum ticks are assumed to transmit $C$. felis $[10,11]$, but more recent studies point to $A$. americanum as the primary definitive host and tick vector of $C$. felis [10, 12-14]. The main natural reservoir of $C$. felis in the USA is considered to be the bobcat (Lynx rufus). Infections in this species are usually subclinical $[11,15-17]$, but fatal disease has been described in bobcats, tigers and lions [15, 18-21].

In recent years, Cytauxzoon spp. infections with a species molecularly distinct from $C$. felis have been reported in domestic cats in a number of European countries, including Italy, France, Spain, Portugal and, most recently, Switzerland and Germany [22-30]. There have also been reports of infections with Cytauxzoon spp. in wild felids in Europe; these include the European wildcat in Italy, Romania, Germany, Luxembourg, Bosnia and Herzegovina [31-34], the Iberian lynx in Spain [35] and the Eurasian lynx in Romania, Czech Republic and Switzerland [32, 34] (MLM, personal communication). Similar to how bobcats serve as the primary reservoir of $C$. felis in the USA [3], the Eurasian and the Iberian lynx may play a role as reservoirs of Cytauxzoon spp. in Europe. The role of the European wildcat in the transmission cycle of Cytauxzoon spp. is unclear, and the tick vector involved in the transmission of Cytauxzoon spp. in Europe has not yet been identified.

Based on sequencing of the $18 S$ rRNA gene, Cytauxzoon spp. isolates from Europe are phylogenetically related but distinct from $C$. felis and most closely related to Cytauxzoon manul from the Pallas' cat [36, 37]. More recently, Panait et al. [34] suggested that three distinct species of Cytauxzoon exist in European wild felids, based on results of phylogenetic analyses of the two mitochondrial genes cytochrome $b(C y t B)$ and cytochrome $c$ oxidase subunit I (COI) from Cytauxzoon spp. isolates obtained from European wildcats and Eurasian lynx from different European countries. One species predominated (Cytauxzoon europaeus), while the two other species were rare and found only in seven (Cytauxzoon otrantorum) and one (Cytauxzoon banethi) European wildcats in Romania, respectively [34]. Whether these species also occur in domestic cats is currently unknown.

Cytauxzoon spp. infections in domestic cats in Europe are generally considered to be less severe than C. felis infections. Indeed, in one study, $22.9 \%$ of the investigated cats in northeast Italy tested PCR positive for Cytauxzoon spp. even though most of these cats appeared to be clinically healthy [23]. Nevertheless, symptomatic and fatal Cytauxzoon spp. infections have been described in domestic cats in Europe [22, 23, 26, 27, 29], with these cats presenting with lethargy, anorexia, weight loss, pyrexia, pale mucous membranes, diarrhoea, vomiting and pleural or peritoneal effusion; however, concomitant diseases were not always excluded [23, 26].

Recently, we reported Cytauxzoon spp. infection for the first time in Switzerland in five domestic cats [29]. All infected cats were from the northwest and west of Switzerland, regions close to the French border. Three infected kittens from the same litter presented with moderate to severe anaemia. The kittens recovered following treatment with atovaquone and azithromycin but developed long-term asymptomatic parasitaemia. One adult cat with non-regenerative anaemia experienced accidental transmission of Cytauxzoon spp. through a blood transfusion from an asymptomatically infected cat.

The recent discovery and expanding geographical distribution of Cytauxzoon spp. in domestic cats and wild felids in Europe suggest that Cytauxzoon spp. infection is an emerging tick-borne disease, but this hypothesis has not been investigated. Following our initial report of Cytauxzoon spp. infection in cats in west and northwest Switzerland in 2014/2015, we discovered an additional six cases in domestic cats in Switzerland that were diagnosed in 2019 (study A); these cats originated from two households in central Switzerland. This finding prompted us to further elucidate the epidemiology and emerging nature of Cytauxzoon spp. infection in Switzerland. For this purpose, we conducted a countrywide survey in privately owned cats from each canton (region) in Switzerland (study B) and investigated the frequency 
of infection in suspected high-risk populations, such as anaemic cats in Switzerland and stray cats in northwestern Switzerland (study C). To address the question of whether Cytauxzoon spp. infection has newly emerged in domestic and wild felids in central Europe, we tested DNA from blood of domestic cats and European wildcats collected in 2003 and 1995-1996, respectively, for the presence of the organism (study D). All Cytauxzoon spp. isolates were subjected to $18 \mathrm{~S}$ rRNA and mitochondrial gene amplification in order to perform in-depth phylogenetic analyses and resolve the genetic relationship of the Cytauxzoon spp. isolates from domestic and wild felids.

\section{Methods}

\section{Study design}

The present study comprised four substudies (studies A-D). In study A, we reported the occurrence of Cytauxzoon spp. infection in six cats from two households in central Switzerland. Study B addressed the countrywide occurrence of Cytauxzoon spp. infection in domestic cats in Switzerland. In study C, we investigated Cytauxzoon spp. infection in suspected high-risk populations, i.e. stray cats in a hotspot region in Switzerland and anaemic cats in Switzerland. Study D addressed the potential emerging nature of Cytauxzoon spp. infections in domestic cats and wild felids in central Europe. Phylogenetic analysis of the Cytauxzoon spp. isolates was performed based upon sequencing of the $18 \mathrm{~S}$ rRNA gene and two mitochondrial genes $(C y t B$ and $C O I)$. Samples for studies $B$ and $D$ and the samples from stray cats for study $C$ were available from previous studies [38-41].

\section{Sample characteristics}

In study A, 13 cats were tested for Cytauxzoon spp. infection (Table 1). The diagnostic services of the Clinical Laboratory, Vetsuisse Faculty Zurich first identified three cats infected with Cytauxzoon spp. in a household in central Switzerland in February-March 2019 (Canton Aargau, household 1) and subsequently in three cats in May 2019 in a neighbouring household in the same village (household 2); in household 2, seven cats subsequently underwent diagnostic testing. Data on the place and region of origin, age, sex and general health status were available for all cats from households 1 and 2 (Additional file 1: Table S1), and all cats were tested for Cytauxzoon spp. and feline leukaemia virus (FeLV) provirus by realtime TaqMan quantitative PCR (qPCR) and for feline immunodeficiency virus (FIV) by western blot (Table 2; Additional file 1: Table S1) [42, 43].

One cat from household 1 underwent a complete necropsy and subsequent histological examination of selected organs (heart, lungs, liver, pancreas, kidney, spleen, lymph nodes) at the Institute of Veterinary
Pathology, Vetsuisse Faculty Zurich, Switzerland. Sections $(3-4 \mu \mathrm{m})$ from formalin-fixed, paraffin-embedded tissue specimens were routinely stained with haematoxylin/eosin and Giemsa. Sections from the lung were also subjected to immunohistological staining for feline CD18 to highlight monocytes/macrophages [44]. Cytological specimens of the pleural effusion were examined, and real-time TaqMan qPCR for Cytauxzoon spp. was performed on blood, pleural fluid, lung, bone marrow, liver, kidney, spleen and pancreas at the Clinical Laboratory, Vetsuisse Faculty Zurich.

Study B comprised 881 total nucleic acid (TNA) samples extracted from ethylenediaminetetraacetic acid (EDTA)-anticoagulated blood of domestic cats presented to private veterinarians for diagnostic purposes (Table 1). The samples had been collected as part of a FeLV prevalence study between September 2013 and April 2016; information on these samples has been published in detail $[38,39]$. Data on place and region (canton) of origin and sex were available for most cats (Table 1). FeLV provirus real-time TaqMan qPCR results were available from the previous study (Table 2) [39]. The quality and quantity of the TNA samples was assessed using a feline albumin real-time TaqMan qPCR assay [45], and only samples with a cycle threshold $(\mathrm{CT})$ value $<30$ were included.

In study C, the frequency of Cytauxzoon spp. infection in suspected high-risk populations was assessed by testing TNA extracted from EDTA-anticoagulated blood of 501 anaemic cats from Switzerland; blood samples were sent for diagnostic purposes to the Clinical Laboratory, Vetsuisse Faculty Zurich between 2019 and 2021 (Table 1). The samples originated from 16 cantons in Switzerland (Table 1) and included 33 cats from the canton of Aargau, where the two affected households from study part A were located; one cat came from the western part of Switzerland (canton of Vaud), where the remaining Cytauxzoon spp. cases in Switzerland have been documented [29]. In addition, TNA samples were included from EDTA-anticoagulated blood of 91 stray cats collected during a trap-neuter-release programme in August and October 2014 in a known hotspot region in northwestern Switzerland in the canton of Jura (Table 1); details of these samples have been published [38]. The sampled cats originated from 19 different villages in the canton of Jura in northwestern Switzerland. The samples had already been analysed for Felis catus gammaherpesvirus (FcaGHV) in a previous study [38], and data on sex and FeLV status were available for most animals (Tables 1,2). All stray cat TNA samples were evaluated using a feline albumin real-time TaqMan qPCR assay [45], and only samples with a CT $<30$ were included. 


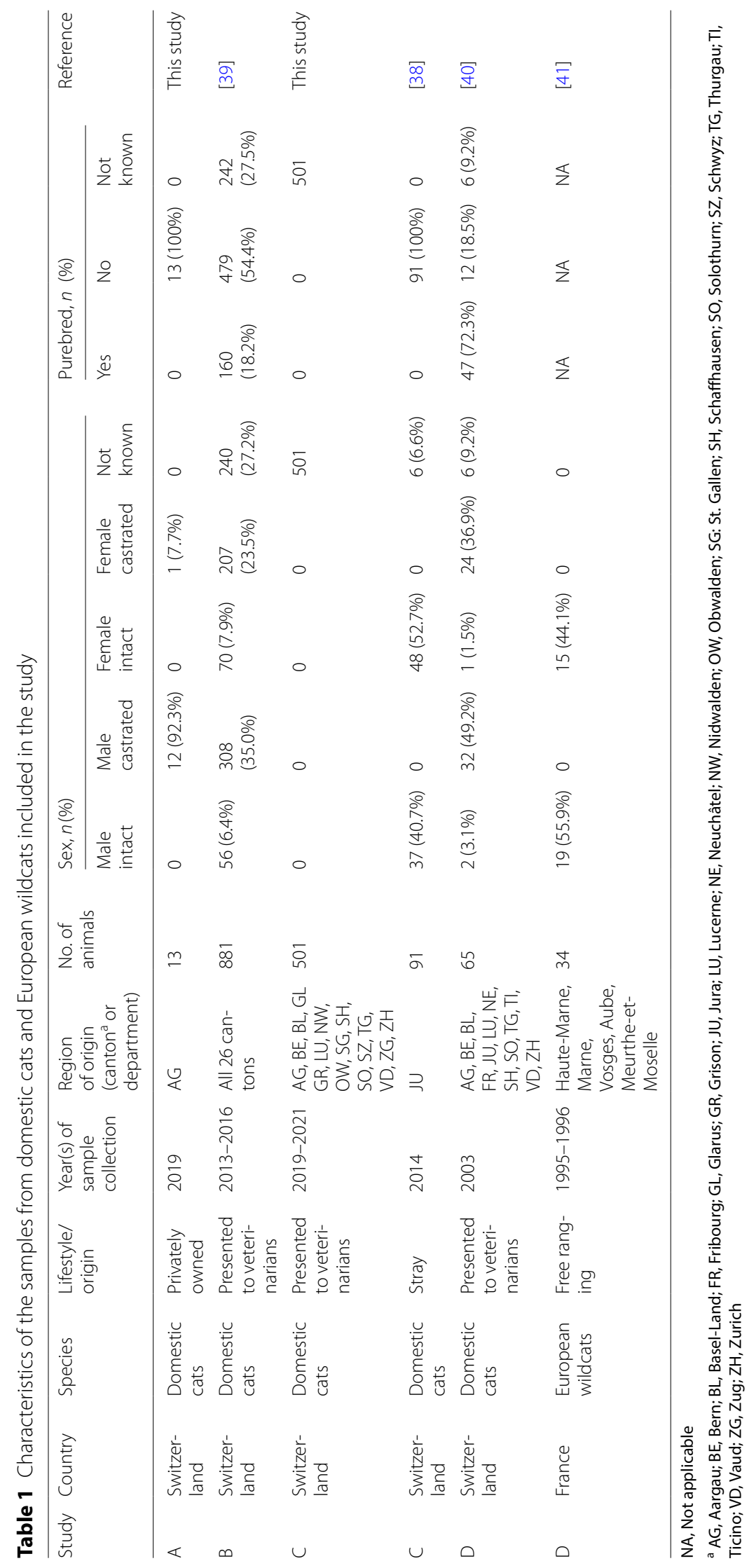




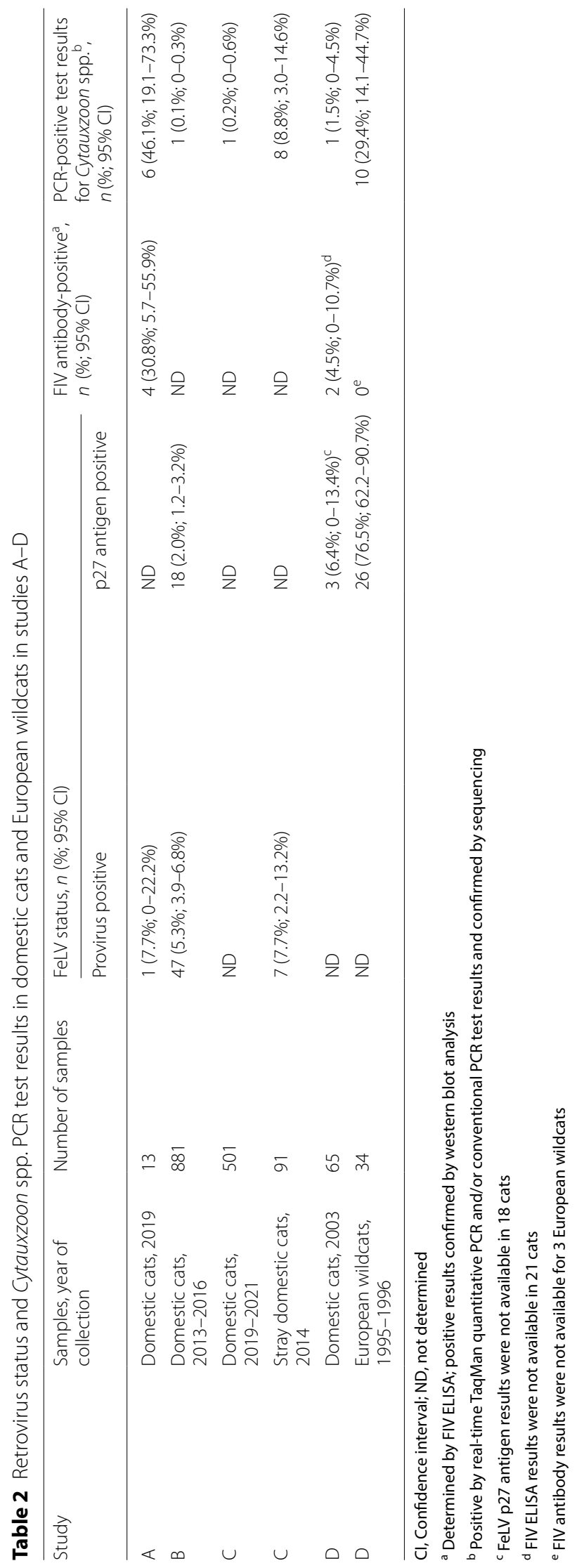


In study D, the emerging nature of Cytauxzoon spp. infection was investigated by testing DNA samples from EDTA-anticoagulated blood of 65 privately-owned cats from Switzerland sampled in 2003 (Table 1). The samples were part of a larger set of feline blood samples previously tested for feline haemoplasmas [40]. The cats originated from the south $(n=8)$, the northwest and west $(n=9)$, and central, eastern and northern Switzerland $(n=48$, Fig. 1a). Furthermore, DNA was included from the blood of 34 European wildcats collected between March 1995 and October 1996 for an unrelated study (Table 1) [41]; the samples originated from six different departments in eastern France (Fig. 1b). Data on place and region of origin, age/age group, sex and retrovirus status were available for most of the included cats and wild felids (Tables 1 , 2 ). The quality and quantity of TNA were evaluated in $47 / 65$ domestic cat samples using the feline albumin realtime TaqMan qPCR assay [45].

\section{Haematology and blood biochemistry}

The results from haematology and blood biochemistry analyses were available for five cats infected with Cytauxzoon spp. from households 1 and 2 (Additional file 2: Table S2; Additional file 3: Table S3); one cat was tested twice, at initial diagnosis (March 2019) and at the time of euthanasia (May 2019; Additional file 2: Table S2; Additional file 3: Table S3). Haematology and blood biochemistry analyses were performed at the Clinical Laboratory, Vetsuisse Faculty, University of Zurich on a Sysmex XT2000iV (Sysmex Corporation, Kobe, Japan) [46] and a Cobas C 501 instrument (Roche Diagnostics AG, Rotkreuz, Switzerland), respectively, or at IDEXX Laboratories (IDEXX Diavet AG, Bäch, Switzerland) using a Sysmex XT-2000iV (Sysmex Corporation) and an AU680 ISE (Beckman Coulter, Inc., Brea CA, USA), respectively. Laboratory (internal validation IDEXX Diavet/IDEXX Ludwigsburg, Ludwigsburg, Germany), and published reference intervals were used [46].

\section{TNA extraction}

Total nucleic acid extraction in study A was performed from $100 \mu \mathrm{l}$ of EDTA-anticoagulated blood using the MagNa Pure LC (Roche Diagnostics AG) and the MagNa Pure LC TNA Isolation Kit (Roche Diagnostics AG) following the manufacturer's instructions. DNA from tissues collected upon necropsy was purified using DNeasy Tissue Kits (Qiagen, Hilden, Germay). A negative control consisting of $100 \mu \mathrm{l}$ of phosphate buffered saline was used with each batch of extraction to monitor for crosscontamination. The TNA and DNA samples were stored at $-80{ }^{\circ} \mathrm{C}$ until PCR analysis was performed.

\section{Diagnostic assays}

The FeLV provirus was detected using a real-time TaqMan qPCR assay as described [42]. FeLV p27 antigenaemia was determined using a published sandwich enzyme-linked immunosorbent assay (ELISA) [47]; a sample signal of $\geq 20 \%$ of the positive control signal was considered to be positive. FIV infection was detected using a western blot [48]. Published real-time TaqMan qPCR assays were used for the detection of feline albumin and $18 S$ ribosomal RNA (rRNA) [45].

All blood DNA and TNA samples of this study were subjected to a Cytauxzoon spp. real-time TaqMan qPCR assay, with the exception of the samples from European wildcats; the latter were tested directly by conventional PCR (see following section). Tissue samples were tested undiluted and at a 1:10 dilution to detect inhibition; if inhibition was present, the $\mathrm{CT}$ value of the diluted sample is given. The primers and probe of the real-time TaqMan qPCR assay were designed to specifically detect a 69-bp fragment of the $18 S$ rRNA sequences of Cytauxzoon spp. found in the NCBI GenBank [29]. All samples with $\mathrm{CT}$ values $<35$ were subsequently subjected to conventional PCR to amplify 219 bp of the $18 S$ rRNA gene of Cytauxzoon spp. [29, 49, 50]. The PCR products were separated in a $2 \%$ agarose gel, and bands of the appropriate size $(219 \mathrm{bp})$ were sequenced in a commercial laboratory (Microsynth AG, Balgach, Switzerland) using the amplification primers. All real-time TaqMan qPCR assays were performed on an ABI 7500 Fast Real-Time PCR system (Applied Biosystems, Thermo Fisher Scientific, Waltham, MA, USA). Positive and negative controls were run with each PCR assay and consisted of DNA from a Cytauxzoon spp. PCR-positive Iberian lynx (confirmed by sequencing) and of nuclease-free water, respectively.

\section{Amplification and sequencing of the $18 \mathrm{~S}$ rRNA, CytB and $\mathrm{COI}$ genes}

All samples that were PCR positive in conventional PCR underwent amplification and sequencing of the $18 S$ rRNA, CytB and COI genes of Cytauxzoon spp. The almost complete $18 S$ rRNA gene was amplified using the forward primer Cytlblynx.23f (5'-GCC ATG CAT GTC TAA GTA TAA GC-3') and the reverse primer Cytlblynx.1659r (5'-CGC GCC TAA CGA ATT AGA AG-3') as previously described [29]. The mitochondrial $C y t B$ and $C O I$ genes were amplified from the same samples using nested PCR assays as previously published [34], with some modifications. Briefly, the reaction mixture contained $5 \mu \mathrm{l}$ of $5 \times$ HF PCR buffer (Finnzymes Oy, Espoo, Finland/BioConcept AG, Allschwil, Switzerland), $500 \mathrm{nM}$ of each primer, $0.2 \mathrm{mM}$ of each deoxynucleotide triphosphate (Sigma-Aldrich, St. Louis, MO, USA), 1 U Phusion High-Fidelity DNA 
Polymerase (Finnzymes Oy) and $5 \mu \mathrm{l}$ template TNA/ DNA, with water added to achieve a a final reaction volume of $25 \mu \mathrm{l}$. The conventional PCR assays were performed on a Biometra T-Personal 48 Thermocycler (Biometra, Gottingen, Germany), using the following cycling programme: $98^{\circ} \mathrm{C}$ for $30 \mathrm{~s} ; 35$ cycles of $98^{\circ} \mathrm{C}$ for 10 s, $53{ }^{\circ} \mathrm{C}$ (Cytaux_cytb_F1/Cytaux_cytb_R3 [CytB]), $55{ }^{\circ} \mathrm{C}$ (Cytaux_cytb_Finn/Cytaux_cytb_Rinn [CytB]), $64{ }^{\circ} \mathrm{C}$ (Th-For2/Piro_mt_R1 [COI]), $60{ }^{\circ} \mathrm{C}$ (Th-For2/ Cytaux_260R [COI]) for $30 \mathrm{~s}, 72{ }^{\circ} \mathrm{C}$ for $1 \mathrm{~min}$; and a final elongation at $72{ }^{\circ} \mathrm{C}$ for $10 \mathrm{~min}$. PCR products were separated in a $1.5 \%$ agarose gel, and bands of the appropriate size were cut and extracted using the MinElute ${ }^{\circledR}$ Gel Extraction Kit (Qiagen) and the PCR products subsequently sequenced.

\section{Genetic and phylogenetic analyses}

Sequences were edited and assembled using Geneious ${ }^{\circledR}$ prime software (Biomatters Limited, Auckland, New Zealand) [51]. Phylogenetic analysis was conducted using MEGA-X [52]. Sequences were aligned to additional reference sequences retrieved from GenBank using the Clustal W algorithm [53]. The phylogenetic tree was inferred using the maximum likelihood method using a distance matrix corrected for nucleotide substitutions based on the Kimura 2-parameter model [54]. The initial tree(s) for the heuristic search were automatically obtained by applying neighbour-joining and BioNJ algorithms to a matrix of pairwise distances estimated using the maximum composite likelihood approach, and then selecting the topology with superior log likelihood value. Codon positions included were 1 st $+2 \mathrm{nd}+3 \mathrm{rd}+$ noncoding. All positions containing gaps and missing data were eliminated. The dataset was resampled 1000 times to generate bootstrap values [55].

\section{Statistical analysis}

For the observed sample prevalences, 95\% confidence intervals (CIs) were calculated using XLSTAT for Microsoft Excel (Addinsoft, NY, USA). The association between Cytauxzoon spp. infection and exposure variables was tested by univariate statistical analysis performed by a chi-squared $\left(\chi^{2}\right)$ test or a Fisher's exact test for small numbers $(n<5)$ using XLSTAT (Addinsoft). $P$ values $<0.05$ were considered to be statistically significant.

\section{Results \\ Study A: Cytauxzoon spp. infection in six cats from two neighbouring households in Switzerland}

After the first report of Cytauxzoon spp. infection in five domestic cats from northwestern and western Switzerland in 2014/1015 [29], the organism was detected again in a cat that was presented to a private veterinary practitioner in February 2019 and subsequently in another five cats based on PCR analysis of TNA extracted from blood samples sent to the Clinical Laboratory, University of Zurich for testing. These cats came from two households in central Switzerland (canton of Aargau), an area where Cytauxzoon spp. had not been previously reported (Table 1; Fig. 1a). The two neighbouring households were located only $182 \mathrm{~m}$ apart. A total of 13 cats were investigated, of which six (46.1\%; 95\% CI: 19.1-73.3\%) were infected with Cytauxzoon spp. (Table 2). All cats were born in the region and had never travelled abroad.

Cytauxzoon spp. infection was first detected in a cat from household 1 that was taken to a private veterinary practitioner due to severe and progressive cellulitis on the right front limb (Additional file 1: Table S1). EDTAanticoagulated blood and serum were taken 1 day after the first presentation and sent to an external reference laboratory (IDEXX Diavet AG) for an extensive checkup. Blood work revealed nonregenerative anaemia, severe leukocytosis, moderate thrombocytopenia, mild hypoalbuminaemia, a mild increase in alanine aminotransferase and a moderate increase in aspartate aminotransaminase (Additional file 2: Table S2, Additional file 3: Table S3). Ring-shaped single or paired inclusion bodies were detected in the blood smear, raising suspicion for the presence of Cytauxzoon spp. organisms. The sample was then transferred to the Clinical Laboratory, University of Zurich, where infection with Cytauxzoon spp. was diagnosed from PCR analyses of TNA extracted from this blood sample and subsequently confirmed by sequencing (Additional file 1: Table S1). Retrovirus testing revealed co-infection with FIV (Additional file 1: Table S1). The cat was euthanised 3 days after first presentation due to poor prognosis; necropsy was not performed.

The two other cats from household 1 were subsequently also tested for Cytauxzoon spp. and retrovirus

\footnotetext{
(See figure on next page.)

Fig. 1 Map of Switzerland (a) and France (b) showing the geographical distribution of the analysed samples. The geographic origin of the cats from study parts A (pentagons), B (circles), C (rhombs: anaemic cats; squares: stray cats) and D (triangles: domestic cats from 2003; stars: European wildcats from 1995-1995) in Switzerland (a) and France (b) are indicated. The colour of the symbols in $\mathbf{a}$ and $\mathbf{b}$ indicate PCR-positive (red) and -negative (green) samples. The size of the symbols in a indicates the number of Cytauxzoon spp. PCR-positive or -negative samples per location. For 11 cats from study B and 30 stray cats from study $C$ the place of origin within the canton was unknown; these samples were allocated to the capital city of the corresponding canton
} 


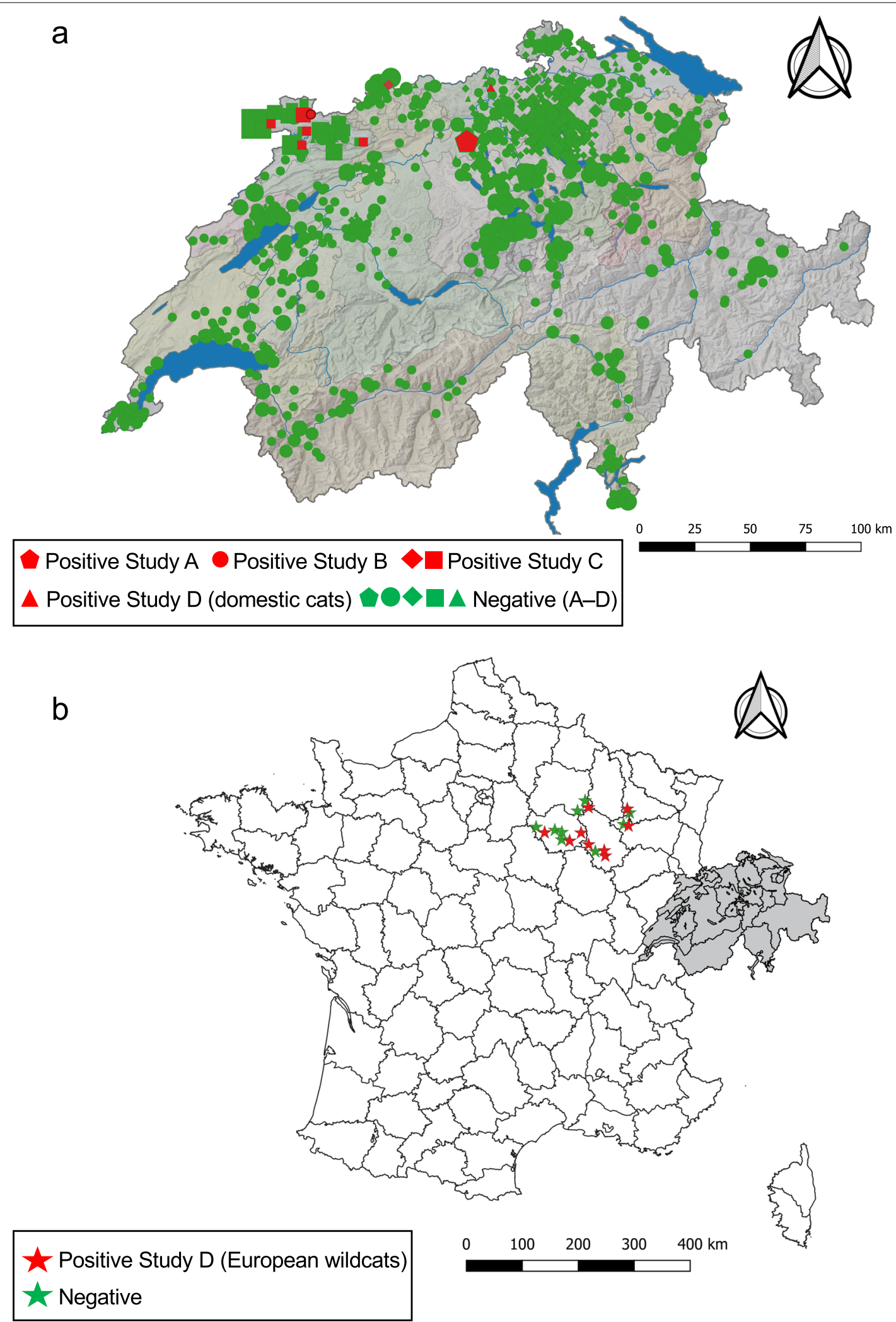

Fig. 1 (See legend on previous page.) 
infection in March 2019. Both cats had positive PCR test results for Cytauxzoon spp., one of which was co-infected with FIV (Additional file 1: Table S1). At the time of diagnosis, both cats were clinically healthy, and had no blood abnormalities except for mild hyperproteinaemia in the FIV-infected cat (Additional file 2: Table S2; Additional file 3: Table S3). One of the two cats developed severe apathy and was euthanised with suspected pyothorax in May 2019 (Additional file 1: Table S1). At the time of euthanasia, the cat showed severe hyperbilirubinaemia in the absence of anaemia (Additional file 2: Table S2; Additional file 3: Table S3). The animal was subjected to a full post-mortem examination, which revealed a severe pyothorax with pulmonary atelectasis and acute fibrinosuppurative pleuritis. A cytological examination of the fluid revealed the presence of filamentous bacteria compatible with Actinomycetaceae, consistent with a diagnosis of actinomycosis/nocardiosis. In addition to pleuritis and atelectasis, histological examination of the lung revealed filamentous Gram-positive bacteria (consistent with Actinomycetaceae) in some capillaries, indicating bacteraemia. Immunohistology for the adhesion molecule CD18 stain identified rare individual large, vacuolated, strongly positive monocytes within capillaries and a few small aggregates of equally positive macrophages within alveolar lumina (Additional file 4: Figure S1a). The spleen and lymph node showed severe lymphocyte depletion, with the splenic red pulp being poorly cellular and the lymph node exhibiting very rare large vacuolated macrophages in the marginal sinuses. The heart, liver and kidney were unremarkable except for very rare, individual large vacuolated cells within the glomerular tufts and one structure suggestive of a large vacuolated monocyte in a myocardial capillary (Additional file 4: Figure S1b). These, as well as the strongly CD18-positive monocytes/ macrophages, were considered indicative of the schizontbearing monocytes/macrophages characteristic for $C$. felis infection [44, 56]; however, particularly in the lungs these could also represent cells activated due to the actinomycosis/nocardiosis. The pleural effusion and all tissue samples examined (spleen, lung, bone marrow, kidney, pancreas, liver) yielded PCR-positive test results for Cytauxzoon spp. but with lower loads (CT values: 24.730.6) compared to blood loads at the time of euthanasia (CT value: 14.7 ).

In April and May 2019, another 10 cats from the neighbouring household 2 were tested for Cytauxzoon spp. and retrovirus infection as part of health checks and because the owner indicated that the cats had aggressive interactions with the cats from household 1 (Additional file 1: Table S1). Of these 10 cats, three tested PCR positive for Cytauxzoon spp. and two were FIV positive; the results for FIV in the other cats were questionable according to the western blot test (detection of only 1 band). Besides some unrelated health problems, the cats were clinically healthy. Blood test results were available for two of the three Cytauxzoon spp-infected animals and revealed only mild and unspecific changes (Additional file 2: Table S2; Additional file 3: Table S3). One cat had mild hyperproteinaemia, but the cat was co-infected with FIV (Additional file 1: Table S1).

\section{Study B: Countrywide survey on Cytauxzoon spp. infection} in domestic cats in Switzerland

Only one of 881 domestic cat samples $(0.1 \%$; $95 \%$ CI: $0-0.3 \%$ ) in the countrywide survey tested PCR positive for Cytauxzoon spp. (Table 2). The positive result was confirmed by sequencing (Additional file 5: Table S4). The cat came from the canton of Jura in the northwest of Switzerland (Fig. 1a). The cat tested negative for FeLV provirus and PCR negative for the three feline haemoplasmas, but had a positive PCR test result for FcaGHV-1 and had a questionable result for FIV by western blot (detection of only 1 band) [38]. No health status data were available for this cat.

\section{Study C: Cytauxzoon spp. infections in stray cats in northwestern Switzerland and in anaemic cats}

Of the 501 anaemic cats, one $(0.2 \%$; $95 \%$ CI: $0-0.6 \%)$ tested Cytauxzoon spp. PCR positive in blood (Table 2). This cat came from northern Switzerland (canton of Basel-Land), where Cytauxzoon spp. had not been previously reported (Fig. 1a) and had been taken to a private veterinary practitioner because of severe anaemia (haematocrit: $12 \%$ ). The cat suffered from chronic renal failure and was co-infected with FIV (Additional file 5: Table S4); no further data or follow-up of this cat were available.

Of the 91 stray cats, eight (8.8\%; 95\% CI 3.0-14.6\%) tested positive for Cytauxzoon spp. (Table 2); positive results were confirmed by sequencing (Additional file 5 : Table S4). The PCR-positive cats were from four different villages (Fig. 1a, Additional file 5: Table S4); the place of origin was unknown for one cat. All eight cats infected with Cytauxzoon spp. tested negative for FeLV provirus (Additional file 5: Table S4); no data were available on the health and FIV status of these cats.

\section{Study D: Cytauxzoon spp. infections in cats and European wildcats sampled in 2003 and 1995-1996, respectively}

To investigate the emerging nature of Cytauxzoon spp. infection in felids, we examined samples collected more than two decades ago from domestic cats in Switzerland $(n=65)$ and European wildcats $(n=34)$ from eastern France. Of the 65 blood DNA samples collected from domestic cats in Switzerland in 2003, one (1.5\%; 95\% 
CI: 0-4.5\%) tested PCR positive for Cytauxzoon spp. (Table 2); the result was confirmed by sequencing (Additional file 5: Table S4). This Cytauxzoon spp.-positive cat was from the canton of Aargau in central Switzerland (Fig. 1a) and tested negative for FeLV by FeLV p27 ELISA and negative for FIV by western blot (Additional file 5: Table S4). The cat had been taken to a veterinary clinic in 2003 because of severe anaemia and icterus. Hepatic lipidosis was diagnosed, and the cat was euthanised due to continuous deterioration. A post-mortem examination was not performed.

Of the 34 European wildcats from eastern France, 10 (29\%; 95\% CI: 14.2-44.7\%) tested PCR positive for Cytauxzoon spp. (Table 2); the results were confirmed by sequencing (Additional file 6: Table S5). Eight of these 10 infected cats tested positive for FeLV p27; none were positive for FIV by western blot (Additional file 6: Table S5). The 10 European wildcats infected with Cytauxzoon spp. were from four different departments in eastern France (Fig. 1b; Additional file 6: Table S5); the places of origin were up to $156 \mathrm{~km}$ apart. Because the samples had been collected from animals hit by cars, no data on the health status of these animals were available.

\section{No association of Cytauxzoon spp. infection with sex and retrovirus status of the cats}

In study $\mathrm{C}$, the prevalence of Cytauxzoon spp. infection was higher in stray cats in the hotspot area $(8.8 \%$; $95 \%$ CI: $3.0-14.6 \%)$ than in domestic cats in the countrywide survey (0.1\%; $95 \%$ CI: $0-0.3 \%)$ and in anaemic cats $(0.2 \%$; 95\%: CI 0-0.6\%; Table 2). Sex and retrovirus infections were not significantly associated with Cytauxzoon spp. infection in any group of samples.

\section{Cytauxzoon spp. sequencing results and phylogenetic analyses}

Sequencing of the almost complete $18 S$ rRNA gene was successful in 18 of the 27 PCR-positive samples; from the remaining nine PCR-positive samples, a shorter fragment (219 bp) corresponding to the amplicon length of the conventional PCR was sequenced (Additional file 1: Table S1; Additional file 5: Table S4; Additional file 6: Table S5). Almost complete $18 S$ rRNA sequences were obtained, in particular from samples with low CT values (<28; Additional file 1: Table S1; Additional file 5: Table S4). Sequence identities in the $18 S$ rRNA gene among isolates in this study ranged from 99.3 to $100 \%$, with no clear differences between sequences from the different groups. Overall, the isolates showed $<96.5 \%$ sequence identity in the $18 S$ rRNA gene when compared to all $C$. felis isolates considered and $>99.2 \%$ identity with all European Cytauxzoon spp. and C. manul isolates considered (Fig. 2), with the exception of one Cytauxzoon spp. isolate from a Japanese brown bear (AB480558) for which the identity ranged between 89.4 and $90.6 \%$; sequence identities were independent of host, collection date and region of origin of the samples.

Sequencing of the mitochondrial genes $C y t B$ and $C O I$ was successful in 24 of the 27 PCR-positive samples. Samples with high CT values ( $>28$ ) could not be amplified; these included the one PCR-positive domestic cat in the countrywide survey (study B) and the PCR-positive cat sample from 2003 (study D). Sequence identities in the mitochondrial genes among isolates of this study ranged from $98.7 \%(C y t B)$ and $98.6 \%(C O I)$ to $100 \%$, with no clear differences between sequences from different study groups. Overall, the study isolates showed $<62.1 \%$ and $78.6 \%$ sequence identities in the $C y t B$ and $C O I$ genes, respectively, with published $C$. felis isolates (Figs. 3, 4). Among the European Cytauxzoon spp. isolates, the mitochondrial genes showed highest sequence identities with those of Cytauxzoon europaeus (CytB: 98.6-100\%; COI: 98.6-100\%), followed by those of Cytauxzoon otrantorum (CytB: 89.6-90.2\%; COI: 94.1-95.1\%) and Cytauxzoon banethi (CytB: 76.1-77.8\%; COI: 88.1-88.8\%).

Phylogenetic analysis based on the $18 S$ rRNA gene showed that the isolates of this study clustered with other European Cytauxzoon spp. isolates from domestic and wild felids, and with C. manul from a Pallas' cat (Fig. 2), and was clearly distinct from the $C$. felis cluster. Phylogenetic analysis based on the $C y t B$ (Fig. 3) and $C O I$ genes (Fig. 4) clearly assigned all sequences of this study to $C$. europaeus, which was recently described in wild felids in Europe [34].

\section{Discussion}

Cytauxzoon spp. infection has been considered a newly emerging disease in domestic and wild felids in central Europe. Our results contradict this assumption and suggest that Cytauxzoon spp. infection has been present in domestic cats and European wildcats in central Europe for more than two decades. Cytauxzoon spp. was first reported in domestic cats in Spain in 2004 [25] and in the Iberian lynx in Spain in 2003 [57], and subsequently in domestic cats and wild felids in different countries across Europe, including Switzerland [22-24, 26-33, 35]. These Cytauxzoon spp. isolates were most closely related to C. manul, which was identified in 2005 in a Pallas' cat imported from Mongolia to Oklahoma [37]. Our results show that Cytauxzoon spp. can be detected in European wildcats in eastern France in 1995-1996 and in the DNA of an EDTA-anticoagulated blood sample collected from a domestic cat in central Switzerland in 2003. It is therefore likely that Cytauxzoon spp. was already present in domestic cats and wild felids in the decades before the 
initial report but was overlooked due to its low pathogenic potential compared to $C$. felis.

All $18 S$ rRNA gene sequences obtained in this study showed high sequence identities and a close phylogenetic relationship, confirming that all isolates belong to the European Cytauxzoon spp. cluster regardless of their origin and are distinct from the $C$. felis group. Sequencing of the mitochondrial genes $C y t B$ and $C O I$ and differentiation of three different species have recently been documented in European wild felids infected with Cytauxzoon spp. in Germany, Italy, Romania, Czech Republic, Bosnia and Herzegovina and Luxembourg [34]. The present study performed these analyses for the first time in Cytauxzoon spp.-infected domestic cats in Europe and revealed that all successfully amplified isolates belonged to C. europaeus, which was the most common species in European wild felids in the above-mentioned study [34]. Our results revealed that all isolates of C. europaeus in the present study shared high sequence identities in the $18 S$ rRNA, CytB and COI gene sequences among each other. This is remarkable considering that isolates from both European wildcats and domestic cats were included and that the European wildcat samples originated from collections made in 1995-1996. The close genetic relationship of these isolates supports a potential exchange of $C$. europaeus between domestic and wild felids and thus a potential role of wild felids as reservoirs for this species. Northwestern and western Switzerland, both documented hotspot regions for Cytauxzoon spp. infections, harbour stable populations of European wildcats and the Eurasian lynx $[58,59]$. Bobcats serve as a major reservoir of $C$. felis in the USA [3]; it is therefore possible that the Eurasian lynx might also play a role as a reservoir of Cytauxzoon spp. in central Europe. In support of this possibility, infection with Cytauxzoon spp. has been detected in Eurasian lynx in Switzerland (MLM, personal communication). The role of the European wildcat as a reservoir of Cytauxzoon spp. is less clear. European wildcats can live in close proximity to and even interbreed with domestic cats [60], which could favour a potential transmission of Cytauxzoon spp. between domestic and wild felids. Testing of recent samples from European wildcats in Switzerland indicates that Cytauxzoon spp. infections are very frequently detected (MLM, personal communication); however, recent samples from animals from eastern France were not available. The pathogenic potential of C. europaeus in European wildcats is unknown. All wildcats included in the present study had been free ranging prior to being hit by a car [41]; thus, no clinical data were available. However, the high prevalence of $C$. europaeus infection in this free-ranging population of European wildcats argues against a high pathogenic potential in this species [31, 32, 34].

The overall prevalence of Cytauxzoon spp. infection in samples collected from Swiss domestic cats between 2013 and 2016 was low (0.1\%). The one cat whose PCR test result was positive for Cytauxzoon spp. in the countrywide survey originated from the canton of Jura in western Switzerland, a previously described hotspot region in which four out of five documented Cytauxzoon spp. infections had occurred in domestic cats [29]. In contrast, a remarkably high frequency of Cytauxzoon spp. infections was identified in two households in central Switzerland (46.1\%) and in stray cats in northwest Switzerland (8.8\%). Stray cats live outdoors and are frequently exposed to potential vectors of Cytauxzoon spp., such as ticks. An association between infection with Cytauxzoon spp. and living in rural areas and outdoor access has recently been documented in domestic cats in Spain and Italy [23, 28]. Similarly, the six Cytauxzoon spp.-infected cats identified in the two households in the canton of Aargau in central Switzerland also lived in a rural area, had outdoor access and interacted aggressively with each other. Accordingly, the prevalence of FIV infection in these cats was remarkably high compared with the generally low prevalence of FIV in Switzerland. Of note, the one cat found to be infected with Cytauxzoon spp. in study $\mathrm{C}$ was also found to be FIV positive. Two cats from household 1 died from bacterial diseases that could have developed as a consequence of aggressive interactions, namely cellulitis on one limb and pyothorax (actinomycosis/nocardiosis). Whether aggressive interactions play a role in the transmission of Cytauxzoon spp. is unknown. However, for the transmission of Cytauxzoon spp., blood-to-blood contact would be required, which is unlikely during cat fights. We have recently documented transmission of Cytauxzoon spp. by blood transfusion, but the question of whether blood transfusion from asymptomatic cats can initiate a full cycle of infection in the recipient remains to be elucidated [29].

\section{(See figure on next page.)}

Fig. 2 Molecular phylogenetic analysis by maximum likelihood method of the 185 rRNA gene. The evolutionary history was inferred by using the maximum likelihood method based on the Kimura 2-parameter model [54]. The tree with the highest log likelihood ( -4029.57$)$ is shown. The percentage of trees in which the associated taxa clustered together is shown next to the branches (values $<60 \%$ are not shown). Initial tree(s) for the heuristic search were obtained automatically by applying the neighbour-joining and BioNJ algorithms to a matrix of pairwise distances estimated using the maximum composite likelihood (MCL) approach, and then selecting the topology with superior log likelihood value. The tree is drawn to scale, with branch lengths measured in the number of substitutions per site. Host and country origin of the sequences are indicated. GenBank accession numbers are shown in brackets. Isolates from this study are written in bold font 
Cytauxzoon europaeus (MT904035) wildcat Italy

Cytauxzoon europaeus (MT904038) wildcat Germany

66 Cytauxzoon europaeus (MT904026) wildcat Italy

- Cytauxzoon europaeus (MT904042) wildcat Luxembourg

Cytauxzoon europaeus (MT904043) wildcat Luxembourg

Cytauxzoon europaeus (MT904039) wildcat Germany

Cytauxzoon europaeus (MW727382) domestic cat (20158 Mimi) Switzerland

Cytauxzoon europaeus (MT904040) wildcat Germany

Cytauxzoon europaeus (MW727381) domestic cat (19832 Flocki) Switzerland

Cytauxzoon europaeus (MW727384) domestic cat (20501 Harry) Switzerland

Cytauxzoon europaeus (MW727385) domestic cat (20870 Carlo) Switzerland

Cytauxzoon europaeus (MW727406) wildcat (33) France

Cytauxzoon europaeus (MW727396) stray domestic cat (53aug) Switzerland

Cytauxzoon sp. (EF094469) Iberian lynx Spain

Cytauxzoon europaeus (MT904036) European Iynx Czech Republic

Cytauxzoon sp. (MF503148) domestic cat (donor) Switzerland

Cytauxzoon europaeus (MW727390) stray domestic cat (7oct) Switzerland

Cytauxzoon sp. (EU622908) domestic cat France

Cytauxzoon europaeus (MT904027) Eurasian lynx Romania

Cytauxzoon europaeus (MT904025) wildcat Bosnia and Herzegovina

Cytauxzoon otrantorum (MT904033) wildcat Romania

Cytauxzoon europaeus (MT904028) wildcat Romania

Cytauxzoon europaeus (MW727397) stray domestic cat (70aug) Switzerland

Cytauxzoon sp. (MF503147) domestic cat (c4d5) Switzerland

Cytauxzoon europaeus (MT904044) wildcat Luxembourg

Cytauxzoon europaeus (MW727402) wildcat (18) France

Cytauxzoon sp. (MF503141) domestic cat (c1d7) Switzerland

Cytauxzoon sp. (KT361080) Eurasian lynx Romania

Cytauxzoon europaeus (MW727398) wildcat (03) France

Cytauxzoon sp. (KT361079) wildcat Romania

Cytauxzoon europaeus (MW727395) stray domestic cat (26aug) Switzerland

Cytauxzoon europaeus (MT904034) wildcat Italy

- Cytauxzoon sp. (EF094470) Iberian Iynx Spain

61 Cytauxzoon europaeus (MW727389) domestic cat (UZH JU2 348) Switzerland

Cytauxzoon europaeus (MW727404) wildcat (23) France

Cytauxzoon europaeus (MW727394) stray domestic cat (20aug) Switzerland

Cytauxzoon europaeus (MT904041) wildcat Germany

Cytauxzoon sp. (AY496273) Iberian lynx Spain

Cytauxzoon sp (MF503143) domestic cat (c2d0) Switzerland

Cytauxzoon europaeus (MW727391) stray domestic cat (13aug) Switzerland

Cytauxzoon europaeus (MW727392) stray domestic cat (18aug) Switzerland

Cytauxzoon europaeus (MW727383) domestic cat (20159 Momo) Switzerland

Cytauxzoon europaeus (MW727387) domestic cat (22726 Grisou) Switzerland

Cytauxzoon sp. (KX881967) domestic cat France

Cytauxzoon sp. (MF503145) domestic cat (c3d0) Switzerland

100

- Cytauxzoon sp. (EF094468) Iberian lynx Spain

- Cytauxzoon otrantorum (MT904030) wildcat Romania

Cytauxzoon otrantorum (MT904031) wildcat Romania

Cytauxzoon banethi (MT904029) wildcat Romania

L Cytauxzoon manul (AY485690) Pallas cat Mongolia

Cytauxzoon sp. (AF531418) Pallas cat Mongolia

- Cytauxzoon sp. (AB480558) brown bear Japan

- Uncultured Cytauxzoon (KM025200) Meerkat South Africa

[Cytauxzoon felis (GU903911) ocelot Brasil

100 Cytauxzoon felis (MT904032) wildcat USA

${ }_{87}$ Cytauxzoon felis (L19080) domestic cat South Africa (passaged from USA)

87 [ Cytauxzoon felis (AY531524) domestic cat USA

Cytauxzoon felis (AF399930) domestic cat USA

Cytauxzoon felis (MT904037) wildcat USA

Cytauxzoon felis (AY679105) domestic cat USA

Theileria annulata (JQ743634)

Fig. 2 (See legend on previous page.) 


\begin{tabular}{|c|c|}
\hline $\begin{array}{l}\text { Fig. } 3 \text { Molecular phylogenetic analysis by maximum } \\
\text { using the maximum likelihood method based on the } \\
\text { The percentage of trees in which the associated taxa } \\
\text { for the heuristic search were obtained automatically } \\
\text { estimated using the } \mathrm{MCL} \text { approach, and then selecti } \\
\text { lengths measured in the number of substitutions pe } \\
\text { shown in brackets. Isolates from this study are writte }\end{array}$ & 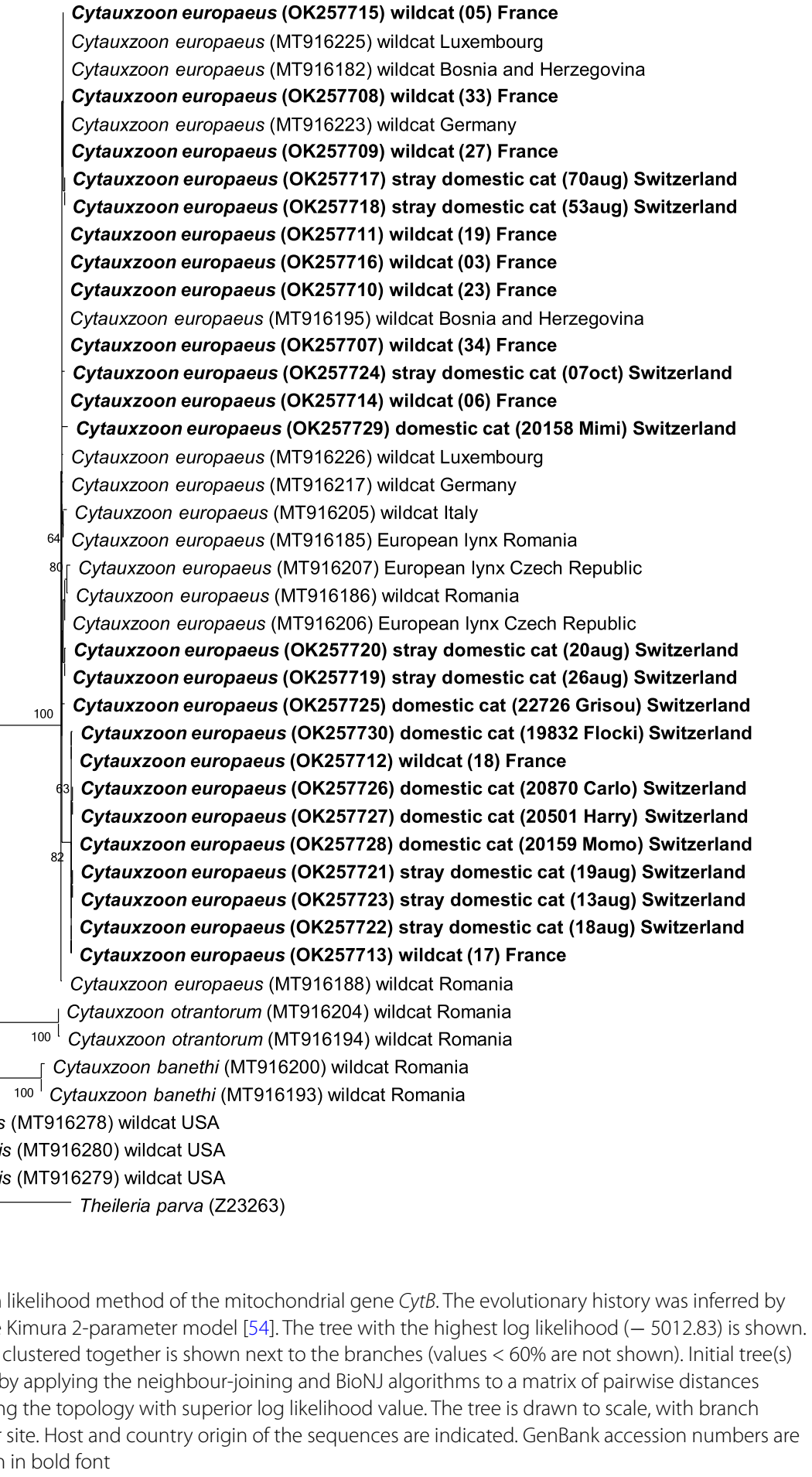 \\
\hline
\end{tabular}

Most of the cats in this study which were infected with Cytauxzoon spp. showed no obvious signs of disease. The schizogonous phase of $C$. felis has been associated with the development of severe clinical disease in domestic cats [3], but schizonts have never been documented in European domestic cats or wild felids infected with Cytauxzoon spp. [22, 23, 27]. In the necropsied cat from household 1 , we observed very rare enlarged, vacuolated 


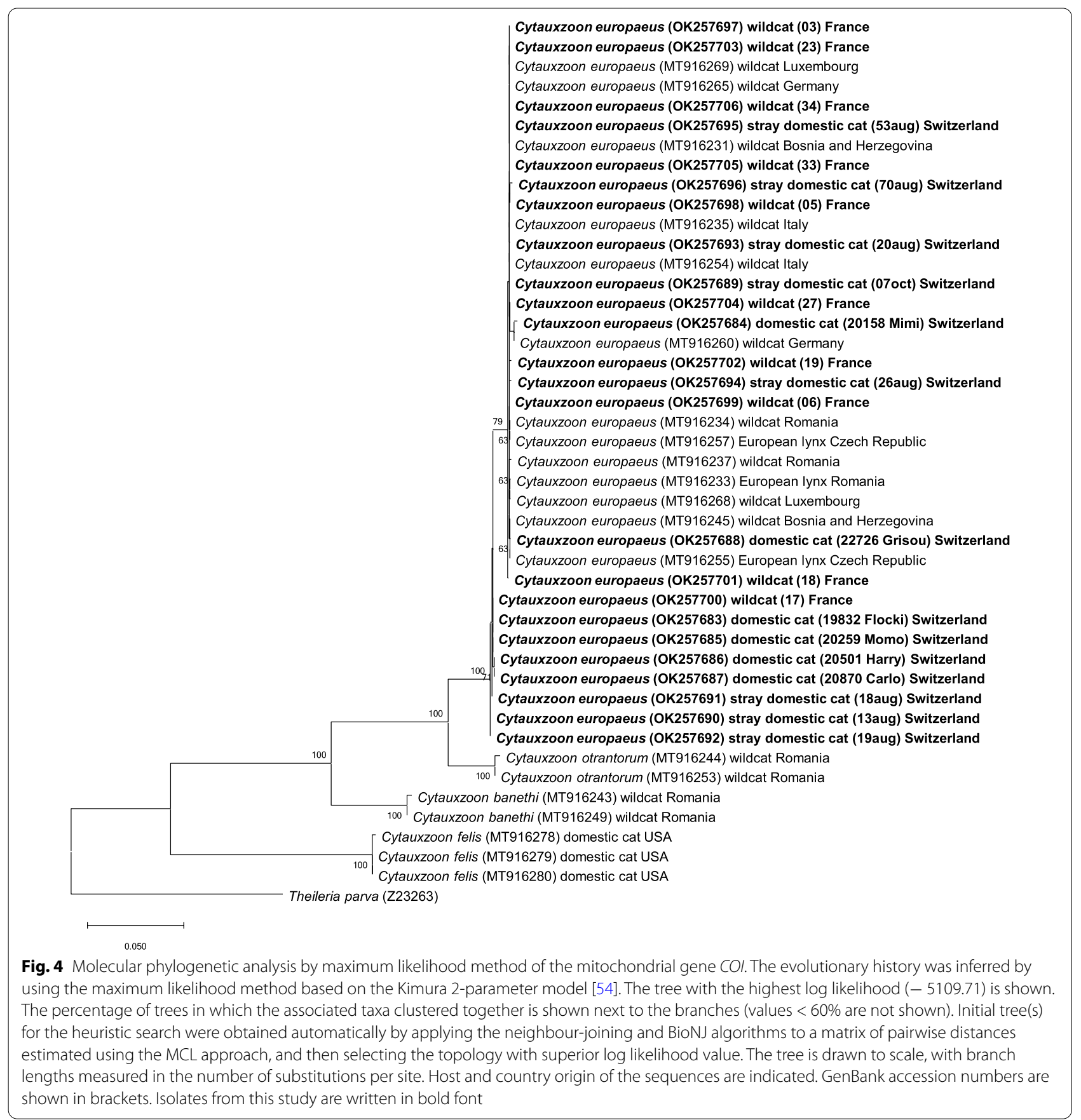

monocytes in capillaries as well as a few aggregates of similarly enlarged macrophages within alveoli that were also strongly CD18 positive, as previously reported for C. felis [44]; considering that they were observed in organs that had tested PCR positive for Cytauxzoon spp., these results could be interpreted with caution as monocytes/macrophages enlarged by schizonts. To confirm this interpretation, further methodological in situ approaches, such as an in situ hybridization with probes specific for Cytauxzoon spp. would be required [61].

The present study also expands the geographic range of Cytauxzoon spp. and provides new insights into the frequency of Cytauxzoon spp. infection in domestic cats in Switzerland. In a previous study we documented the canton of Jura, close to the French border in the northwest of Switzerland, as a hotspot of Cytauxzoon spp. infection, 
where four out of five cats infected with Cytauxzoon spp. originated and three of the five cats were siblings from a stray queen [29]. The second infection hotspot was located in central Switzerland (canton of Aargau) and comprised two neighbouring multi-cat households. These results suggest that Cytauxzoon spp. hotspots exist in different parts of the country. Of note, the cat sample from 2003 that tested positive for Cytauxzoon spp. also came from this canton, with the sites of origin only $30 \mathrm{~km}$ apart. In study $\mathrm{C}$, one infected cat was documented in northern Switzerland, in the canton of Basel-Land, close to the German border. Infection with Cytauxzoon spp. was also recently reported in a 6-year-old domestic cat from Saarlouis (Saarland), Germany that presented with anorexia, lethargy and weight loss [30].

Interestingly, Cytauxzoon spp. infections were not identified in the eastern and southern part of Switzerland. No samples from stray cats from southern and eastern Switzerland were included in our study, which might have contributed to the lack of Cytauxzoon spp. detection in these regions. The geographic differences could also be due to the presence or absence of European wildcats, a potential reservoir of Cytauxzoon spp. in Europe. European wildcats are frequently present in the west and northwest regions of Switzerland, but are absent from southern and eastern Switzerland [62]. However, the Eurasian lynx, also a potential reservoir host, is present in all parts of Switzerland [62]. Another explanation for the absence of Cytauxzoon spp. infections in eastern and southern Switzerland could be the variable distribution of potential tick vectors within the country. For C. felis, Dermacentor variabilis and Amblyomma americanum ticks are known vectors for transmission [10, 12-14], but neither tick species is present in central Europe. Ixodes ricinus is by far the most common tick species in Switzerland and present in all parts of the country [62], whereas D. marginatus and Haemaphysalis punctata are mainly reported in southern and western Switzerland [62]. Dermacentor reticulatus and Rhipicephalus sanguineus ticks are rare and mainly found in western Switzerland [62]. The identification of the vector tick species for Cytauxzoon spp. in Europe is of pivotal interest to further elucidate the distribution and life-cycle of this pathogen in domestic and wild felids.

\section{Conclusions}

Our study raises the question of whether Cytauxzoon spp. is newly emerging in central Europe given that a large proportion of the European wildcat samples collected in 1995 and 1996 in France and a sample from a domestic cat in Switzerland collected in 2003 tested positive for this organism. Mitochondrial gene sequencing revealed that all successfully sequenced Cytauxzoon spp. isolates from domestic cats and European wildcats belonged to $C$. europaeus, which was recently shown to be the most common Cytauxzoon spp. species in European wild felids. The overall prevalence of Cytauxzoon spp. infection in domestic cats in Switzerland was found to be low, but a high prevalence was observed in stray cats and in privately owned cats in hotspot areas, i.e. northwest and central Switzerland. Future studies should address potential vectors and the life-cycle of Cytauxzoon spp. in domestic and wild felids in Europe.

\section{Abbreviations}

COl: Cytochrome c oxidase subunit l; CT: Cycle threshold; CytB: Cytochrome b; EDTA: Ethylenediaminetetraacetic acid; ELISA: Enzyme-linked immunosorbent assay; FcaGHV1: Felis catus gammaherpesvirus 1; FeLV: Feline leukaemia virus; FIV: Feline immunodeficiency virus; rRNA: Ribosomal RNA; TNA: Total nucleic acid.

\section{Supplementary Information}

The online version contains supplementary material available at https://doi. org/10.1186/s13071-021-05111-8.

Additional file 1: Table S1. Origin, signalment, health status, retrovirus status and Cytauxzoon spp. results in the 13 domestic cats investigated in study A.

Additional file 2: Table S2. Haematological analyses of 5 cats infected with Cytauxzoon spp. from households 1 and 2. Results outside the reference interval are shown in bold font.

Additional file 3: Table S3. Biochemistry analyses of 5 cats infected with Cytauxzoon spp. from households 1 and 2. Results outside the reference interval are shown in bold font.

Additional file 4: Figure S1. Cytauxzoon spp.-positive cat from household 1 (study A), euthanised due to pyothorax consistent with actinomycosis/ nocardiosis. Histological and immunohistological features indicative of monocytes/macrophages with schizonts. a Lung. Left: focal aggregate of strongly CD18-positive large macrophages in an alveolus (arrow); right: capillary with strongly CD18-positive large monocyte (arrowhead). Alveolar macrophages are also CD18-positive. Immunohistology, haematoxylin counterstain. Bars: $20 \mu \mathrm{m}$. b Evidence of large vacuolated monocytes. Kidney, glomerulum with structure indicative of large vacuolated monocyte. Haematoxylin eosin stain. Bar: $20 \mu \mathrm{m}$. Right insert: Giemsa-stained section of a glomerulum with structure indicative of large vacuolated monocyte. Bar: $10 \mu \mathrm{m}$. Left inset: myocardial vessel with structure indicative of large vacuolated monocyte. Haematoxylin eosin stain. Bar: $10 \mu \mathrm{m}$.

Additional file 5: Table S4. Origin, signalment, health status and retrovirus status of the Cytauxzoon spp.-infected domestic cats in studies B-D.

Additional file 6: Table S5. Origin, signalment and retrovirus status of the Cytauxzoon spp.-infected European wildcats in study D.

\section{Acknowledgements}

The authors thank the animal rescue organization NetAP, and especially E. Geisser, E. Clavadetscher and H. Steinlin, for providing access to the samples and data from the stray cats. A special thanks goes to E. Bönzli, B. Pineroli, T. Meili, E. Goenczi, S. Childers and the technicians of the Clinical Laboratory for their excellent laboratory assistance. We are grateful to all veterinarians and especially to L. Wach-Gygax for supporting diagnostic work-up and contributing samples to the study. The laboratory work was performed using the logistics of the Center for Clinical Studies, Vetsuisse Faculty, University of Zurich. 


\section{Authors' contributions}

$\mathrm{RHL}$ and BW conceived the study, and $\mathrm{RHL}, \mathrm{BW}$ and $\mathrm{UOG}$ were responsible for the study coordination. $C C, J H, A H, D S, W R$ and UOG were responsible for the sample and data collections from catss. MLM was responsible for all molecular laboratory aspects and sequence data analysis. BR and UOG were responsible for the haematology, cytology and blood biochemistry tests. AK contributed the pathological examinations. BW drafted the manuscript. BW, RHL, MLM, JH, AK, AH and UOG edited the manuscript. All of the authors read and approved the final manuscript.

\section{Funding}

Not applicable.

\section{Availability of data and materials}

The datasets supporting the conclusions of this article are included within the article. Nucleotide sequences obtained in this study have been submitted to GenBank: 18S rRNA genes (MW727381-MW727407), CytB genes (OK257707OK257730) and COI genes (OK257683-OK257706).

\section{Declarations}

\section{Ethics approval and consent to participate}

Samples from the 13 cats in households 1 and 2 (study A) were collected by private practitioners as part of routine diagnostic work-up performed and submitted to the Clinical Laboratory, Vetsuisse Faculty, University of Zurich. Samples from the 91 stray cats (study C) were collected by veterinarians during a trap-neuter-release programme for diagnostic purposes [38]; no study-specific procedures were performed on the cats. Samples from the 881 cats from all cantons in Switzerland (study B) [38, 39] and from the 501 anaemic cats (study C) were residual material from samples submitted for routine diagnostic purposes; no additional volumes or samples were collected for the current study. The post-mortem examination on the one ill cat was undertaken with informed owner consent.

\section{Consent for publication}

Not applicable.

\section{Competing interests}

The authors declare that they have no competing interests.

\section{Author details}

${ }^{1}$ Clinic for Small Animal Internal Medicine, Department for Small Animals, Vetsuisse Faculty, University of Zurich, Zurich, Switzerland. ${ }^{2}$ Clinical Laboratory, Department of Clinical Diagnostics and Services, Vetsuisse Faculty, University of Zurich, Zurich, Switzerland. ${ }^{3}$ Center for Clinical Studies, Vetsuisse Faculty, University of Zurich, Zurich, Switzerland. ${ }^{4}$ IDEXX Diavet Laboratories, Bäch, Switzerland. ${ }^{5}$ Institute of Veterinary Pathology, Vetsuisse Faculty, University of Zurich, Zurich, Switzerland. ${ }^{6}$ Clinical Diagnostic Laboratory, Department of Clinical Veterinary Medicine, Vetsuisse Faculty, University of Bern, Bern, Switzerland. ${ }^{2}$ Labor Am Zugersee, Hünenberg, Switzerland. ${ }^{8}$ Labor Zentral, Geuensee, Switzerland.

Received: 25 May 2021 Accepted: 25 November 2021

Published online: 08 January 2022

\section{References}

1. Brown HM, Berghaus RD, Latimer KS, Britt JO, Rakich PM, Peterson DS. Genetic variability of Cytauxzoon felis from 88 infected domestic cats in Arkansas and Georgia. J Vet Diagn Invest. 2009;21:59-63.

2. Shock BC, Birkenheuer AJ, Patton LL, Olfenbuttel C, Beringer J, Grove DM, et al. Variation in the ITS-1 and ITS-2 rRNA genomic regions of Cytauxzoon felis from bobcats and pumas in the eastern United States and comparison with sequences from domestic cats. Vet Parasitol. 2012;190:29-35.

3. Meinkoth JH, Kocan AA. Feline cytauxzoonosis. Vet Clin North Am Small Anim Pract. 2005;35:89-101.

4. Wang JL, Li TT, Liu GH, Zhu XQ, Yao C. Two tales of Cytauxzoon felis infections in domestic cats. Clin Microbiol Rev. 2017;30:861-85.
5. Haber MD, Tucker MD, Marr HS, Levy JK, Burgess J, Lappin MR, et al. The detection of Cytauxzoon felis in apparently healthy free-roaming cats in the USA. Vet Parasitol. 2007;146:316-20.

6. Brown HM, Latimer KS, Erikson LE, Cashwell ME, Britt JO, Peterson DS. Detection of persistent Cytauxzoon felis infection by polymerase chain reaction in three asymptomatic domestic cats. J Vet Diagn Invest. 2008;20:485-8

7. Rizzi TE, Reichard MV, Cohn LA, Birkenheuer AJ, Taylor JD, Meinkoth JH. Prevalence of Cytauxzoon felis infection in healthy cats from enzootic areas in Arkansas, Missouri, and Oklahoma. Parasit Vectors. 2015;8:13.

8. Wagner JE. A fatal cytauxzoonosis-like disease in cats. J Am Vet Med Assoc. 1976;168:585-8.

9. Birkenheuer AJ, Le JA, Valenzisi AM, Tucker MD, Levy MG, Breitschwerdt EB. Cytauxzoon felis infection in cats in the mid-Atlantic states: 34 cases (1998-2004). J Am Vet Med Assoc. 2006;228:568-71.

10. Reichard MV, Edwards AC, Meinkoth JH, Snider TA, Meinkoth KR, Heinz $\mathrm{RE}$, et al. Confirmation of Amblyomma americanum (Acari: Ixodidae) as a vector for Cytauxzoon felis (Piroplasmorida: Theileriidae) to domestic cats. J Med Entomol. 2010;47:890-6.

11. Blouin EF, Kocan AA, Glenn BL, Kocan KM, Hair JA. Transmission of Cytauxzoon felis Kier, 1979 from bobcats, Felis rufus (Schreber), to domestic cats by Dermacentor variabilis (Say). J Wildl Dis. 1984;20:241-2.

12. Allen KE, Thomas JE, Wohltjen ML, Reichard MV. Transmission of Cytauxzoon felis to domestic cats by Amblyomma americanum nymphs. Parasit Vectors. 2019:12:28.

13. Reichard MV, Baum KA, Cadenhead SC, Snider TA. Temporal occurrence and environmental risk factors associated with cytauxzoonosis in domestic cats. Vet Parasitol. 2008;152:314-20.

14. Reichard MV, Meinkoth JH, Edwards AC, Snider TA, Kocan KM, Blouin EF, et al. Transmission of Cytauxzoon felis to a domestic cat by Amblyomma americanum. Vet Parasitol. 2009;161:110-5.

15. Blouin EF, Kocan AA, Kocan KM, Hair J. Evidence of a limited schizogonous cycle for Cytauxzoon felis in bobcats following exposure to infected ticks. J Wildl Dis. 1987;23:499-501.

16. Glenn BL, Kocan AA, Blouin EF. Cytauxzoonosis in bobcats. J Am Vet Med Assoc 1983:183:1155-8.

17. Zieman EA, Nielsen CK, Jimenez FA. Chronic Cytauxzoon felis infections in wild-caught bobcats (Lynx rufus). Vet Parasitol. 2018;252:67-9.

18. Nietfeld JC, Pollock C. Fatal cytauxzoonosis in a free-ranging bobcat (Lynx rufus). J Wildl Dis. 2002:38:607-10.

19. Peixoto PV, Soares CO, Scofield A, Santiago CD, Franca TN, Barros SS Fatal cytauxzoonosis in captive-reared lions in Brazil. Vet Parasitol. 2007;145:383-7.

20. Garner MM, Lung NP, Citino S, Greiner EC, Harvey JW, Homer BL. Fatal cytauxzoonosis in a captive-reared white tiger (Panthera tigris). Vet Pathol. 1996:33:82-6.

21. Jakob W, Wesemeier $\mathrm{HH}$. A fatal infection in a Bengal tiger resembling cytauxzoonosis in domestic cats. J Comp Pathol. 1996;114:439-44.

22. Carli E, Trotta M, Bianchi E, Furlanello T, Caldin M, Pietrobelli M, et al. Cytauxzoon sp. infection in two free ranging young cats: clinicopathological findings, therapy and follow up. Turkiye Parazitol Dergisi. 2014;38:185-9.

23. Carli E, Trotta M, Chinelli R, Drigo M, Sinigoi L, Tosolini P, et al. Cytauxzoon sp. infection in the first endemic focus described in domestic cats in Europe. Vet Parasitol. 2012;183:343-52.

24. Criado-Fornelio A, Buling A, Pingret JL, Etievant M, Boucraut-Baralon C, Alongi $A$, et al. Hemoprotozoa of domestic animals in France: prevalence and molecular characterization. Vet Parasitol. 2009;159:73-6.

25. Criado-Fornelio A, Gonzalez-del-Rio MA, Buling-Sarana A, Barba-Carretero JC. The "expanding universe" of piroplasms. Vet Parasitol. 2004;1 19:337-45.

26. Alho AM, Silva J, Fonseca MJ, Santos F, Nunes C, de Carvalho LM, et al. First report of Cytauxzoon sp. infection in a domestic cat from Portugal. Parasit Vectors. 2016;9:220.

27. Legroux JP, Halos L, Rene-Martellet M, Servonnet M, Pingret JL, Bourdoiseau G, et al. First clinical case report of Cytauxzoon sp. infection in a domestic cat in France. BMC Vet Res. 2017;13:81.

28. Diaz-Reganon D, Villaescusa A, Ayllon T, Rodriguez-Franco F, Baneth G, Calleja-Bueno L, et al. Molecular detection of Hepatozoon spp. and Cytauxzoon sp. in domestic and stray cats from Madrid, Spain. Parasit Vectors. 2017; 10:112 
29. Nentwig A, Meli ML, Schrack J, Reichler IM, Riond B, Gloor C, et al. First report of Cytauxzoon sp. infection in domestic cats in Switzerland: natural and transfusion-transmitted infections. Parasit Vectors. 2018;11:292. https://doi.org/10.1186/s13071-018-2728-5.

30. Panait LC, Stock G, Globokar M, Balzer J, Groth B, Mihalca AD, et al. First report of Cytauxzoon sp. infection in Germany: organism description and molecular confirmation in a domestic cat. Parasitol Res. 2020;119:3005-11.

31. Veronesi F, Ravagnan S, Cerquetella M, Carli E, Olivieri E, Santoro A, et al. First detection of Cytauxzoon spp. infection in European wildcats (Felis silvestris silvestris) of Italy. Ticks Tick Borne Dis. 2016;7:853-8.

32. Gallusova M, Jirsova D, Mihalca AD, Gherman CM, D'Amico G, Qablan MA, et al. Cytauxzoon infections in wild felids from Carpathian-Danubian-Pontic space: further evidence for a different Cytauxzoon species in European felids. J Parasitol. 2016;102:377-80

33. Hodzic A, Alic A, Duscher GG. High diversity of blood-associated parasites and bacteria in European wild cats in Bosnia and Herzegovina: a molecular study. Ticks Tick Borne Dis. 2018:9:589-93.

34. Panait LC, Mihalca AD, Modry D, Jurankova J, lonica AM, Deak G, et al. Three new species of Cytauxzoon in European wild felids. Vet Parasitol. 2021;290:109344

35. Millan J, Naranjo V, Rodriguez A, de la Lastra JM, Mangold AJ, de la Fuente J. Prevalence of infection and 185 rRNA gene sequences of Cytauxzoon species in Iberian lynx (Lynx pardinus) in Spain. Parasitology. 2007;134:995-1001.

36. Ketz-Riley CJ, Reichard MV, Van den Bussche RA, Hoover JP, Meinkoth J, Kocan AA. An intraerythrocytic small piroplasm in wild-caught Pallas's cats (Otocolobus manul) from Mongolia. JWildl Dis. 2003;39:424-30.

37. Reichard MV, Van Den Bussche RA, Meinkoth JH, Hoover JP, Kocan AA A new species of Cytauxzoon from Pallas' cats caught in Mongolia and comments on the systematics and taxonomy of piroplasmids. J Parasitol. 2005;91:420-6.

38. Novacco M, Kohan NR, Stirn M, Meli ML, Diaz-Sanchez AA, Boretti FS, et al. Prevalence, geographic distribution, risk factors and co-infections of feline gammaherpesvirus infections in domestic cats in Switzerland. Viruses. 2019;11:721.

39. Hofmann-Lehmann R, Gonczi E, Riond B, Meli M, Willi B, Howard J, et al. Feline leukemia virus infection: importance and current situation in Switzerland. Schweiz Arch Tierheilkd. 2018;160:95-105.

40. Willi B, Boretti FS, Baumgartner C, Tasker S, Wenger B, Cattori V, et al. Prevalence, risk factor analysis, and follow-up of infections caused by three feline hemoplasma species in cats in Switzerland. J Clin Microbiol. 2006:44:961-9.

41. Leutenegger CM, Hofmann-Lehmann R, Riols C, Liberek M, Worel G, Lups $P$, et al. Viral infections in free-living populations of the European wildcat. J Wildl Dis. 1999;35(4):678-86.

42. Tandon R, Cattori V, Gomes-Keller MA, Meli ML, Golder MC, Lutz H, et al. Quantitation of feline leukaemia virus viral and proviral loads by TaqMan real-time polymerase chain reaction. J Virol Methods. 2005;130:124-32.

43. Lutz H, Arnold P, Hubscher U, Egberink H, Pedersen N, Horzinek MC. Specificity assessment of feline T-lymphotropic lentivirus serology. Zentralbl Veterinarmed B. 1988;35:773-8.

44. Frontera-Acevedo K, Balsone NM, Dugan MA, Makemson CR, Sellers LB, Brown HM, et al. Systemic immune responses in Cytauxzoon felis-infected domestic cats. Am J Vet Res. 2013;74:901-9.

45. Helfer-Hungerbuehler AK, Widmer S, Hofmann-Lehmann R. GAPDH pseudogenes and the quantification of feline genomic DNA equivalents. Mol Biol Int. 2013;2013:587680.

46. Weissenbacher S, Riond B, Hofmann-Lehmann R, Lutz H. Evaluation of a novel haematology analyser for use with feline blood. Vet J. 2011;187(3):381-7.

47. Lutz H, Pedersen NC, Durbin R, Theilen GH. Monoclonal antibodies to three epitopic regions of feline leukemia virus p27 and their use in enzyme-linked immunosorbent assay of p27. J Immunol Methods. 1983;56:209-20

48. Frankenfeld J, Meili T, Meli ML, Riond B, Helfer-Hungerbuehler AK, Bonzli $E$, et al. Decreased sensitivity of the serological detection of feline immunodeficiency virus infection potentially due to imported genetic variants. Viruses. 2019;11:697.

49. Meli ML, Cattori V, Martinez F, Lopez G, Vargas A, Simon MA, et al. Feline leukemia virus and other pathogens as important threats to the survival of the critically endangered Iberian lynx (Lynx pardinus). PLoS ONE. 2009:4:e4744.

50. Tochtermann C, Willi B. Cytauxzoonosis in domestic cats: an unknown disease in Switzerland? Master's thesis. Zurich: Vetsuisse Faculty, University of Zurich; 2015. p. 1-32.

51. Kearse M, Moir R, Wilson A, Stones-Havas S, Cheung M, Sturrock S, et al. Geneious Basic: an integrated and extendable desktop software platform for the organization and analysis of sequence data. Bioinformatics. 2012;28:1647-9.

52. Kumar S, Stecher G, Li M, Knyaz C, Tamura K. MEGA X: molecular evolutionary genetics analysis across computing platforms. Mol Biol Evol. 2018:35:1547-9.

53. Thompson JD, Higgins DG, Gibson TJ. CLUSTAL W: improving the sensitivity of progressive multiple sequence alignment through sequence weighting, position-specific gap penalties and weight matrix choice. Nucleic Acids Res. 1994:22:4673-80.

54. Kimura M. A simple method for estimating evolutionary rates of base substitutions through comparative studies of nucleotide sequences. $J$ Mol Evol. 1980;16:111-20.

55. Felsenstein J. Confidence limits on phylogenies: an approach using the bootstrap. Evolution. 1985;39:783-91.

56. Snider TA, Confer AW, Payton ME. Pulmonary histopathology of Cytauxzoon felis infections in the cat. Vet Pathol. 2010;47:698-702.

57. Luaces I, Aguirre E, Garcia-Montijano M, Velarde J, Tesouro MA, Sanchez $C$, et al. First report of an intraerythrocytic small piroplasm in wild Iberian lynx (Lynx pardinus). J Wildl Dis. 2005;41:810-5.

58. KORA Foundation. Verbreitung des Eurasischen Luchses in der Schweiz. 2018. http://www.kora.ch/index.php?id=195\&L=0\%29serMode\%22. Accessed 17 Sept 2021

59. KORA Foundation. Verbreitungsgebiet der Europäischen Wildkatze in der Schweiz. 2019. http://www.kora.ch/index.php?id=169. Accessed 17 Sept 2021.

60. Hertwig ST, Schweizer M, Stepanow S, Jungnickel A, Böhle U-R, Fischer MS. Regionally high rates of hybridization and introgression in German wildcat populations (Felis silvestris, Carnivora, Felidae). J Zool Syst Evol Res. 2009:47:283-97.

61. Susta L, Torres-Velez F, Zhang J, Brown C. An in situ hybridization and immunohistochemical study of cytauxzoonosis in domestic cats. Vet Pathol. 2009;46:1197-204

62. Schweizerisches Zentrum für die Kartografie der Fauna (SZKF/CSCF). Info fauna, distribution of species. 2021 https://lepus.unine.ch/carto-old/. Accessed 17 Sept 2021

\section{Publisher's Note}

Springer Nature remains neutral with regard to jurisdictional claims in published maps and institutional affiliations.

Ready to submit your research? Choose BMC and benefit from

- fast, convenient online submission

- thorough peer review by experienced researchers in your field

- rapid publication on acceptance

- support for research data, including large and complex data types

- gold Open Access which fosters wider collaboration and increased citations

- maximum visibility for your research: over 100M website views per year

At BMC, research is always in progress.

Learn more biomedcentral.com/submissions 